\title{
Fluid-structure interaction and in vitro analysis of a real bileaflet mitral prosthetic valve to gain insight into Doppler-silent thrombosis
}

Annalisa Dimasi ${ }^{a}$, Daniela Piloni ${ }^{a}$, Laura Spreafico ${ }^{a}, E^{2}$ iliano Votta ${ }^{a}$, Riccardo Vismara ${ }^{a}$, Gianfranco Beniamino Fiore ${ }^{a}$, Masoud Meskin $^{a}$, Laura Fusini ${ }^{b}$, Manuela Muratori ${ }^{b}$, Piero Montorsi ${ }^{b}$, Mauro Pepi $^{\mathrm{b}}$, Alberto Redaelli ${ }^{\mathrm{a}}$

${ }^{a}$ Department of Electronics, Information and Bioengineering, Politecnico di Milano, Via Golgi 39, 20133 Milan, Italy

${ }^{\mathrm{b}}$ Centro Cardiologico Monzino IRCCS, Via Carlo Parea 4, 20138 Milan, Italy

\section{Corresponding author:}

Alberto Redaelli

Department of Electronics, Information and Bioengineering

Via Golgi 39, 20133 Milano Italy

email: alberto.redaelli@polimi.it

tel. +390223993375

fax. +390223993360

Keywords: Prosthetic valve thrombosis, Doppler silent thrombosis, Bileaflet mechanical valves, Computational fluid dynamics, Fluid-structure interaction.

Word count: 4872 (without references) 


\section{ABSTRACT}

Prosthetic valve thrombosis (PVT) is a serious complication affecting prosthetic heart valves. The transvalvular mean pressure gradient (MPG) derived by Doppler echocardiography is a crucial index to diagnose PVT, but may result in false negatives mainly in case of bileaflet mechanical valves (BMVs) in mitral position. This may happen because MPG estimation relies on simplifying assumptions on the transvalvular fluid dynamics or because Doppler examination is manual and operator-dependent. A deeper understanding of these issues may allow for improving PVT diagnosis and management.

To this aim, we used in vitro and fluid-structure interaction (FSI) modeling to simulate the function of a real mitral BMV in different configurations: normally functioning and stenotic with symmetric and completely asymmetric leaflet opening, respectively. In each condition, the MPG was measured in vitro, computed directly from FSI simulations and derived from the corresponding velocity field through a Doppler-like post-processing approach. Following verification vs. in vitro data, MPG computational data were analyzed to test their dependency on the severity of fluid-dynamic derangements and on the measurement site.

Computed MPG clearly discriminated between normally functioning and stenotic configurations. They did not depend markedly on the site of measurement, yet differences below $3 \mathrm{mmHg}$ were found between MPG values at the central and lateral orifices of the BMV. This evidence suggests a mild uncertainty of the Doppler-based evaluation of the MPG due to probe positioning, which yet may lead to false negatives when analyzing subjects with almost normal MPG. 


\section{INTRODUCTION}

Prosthetic valve thrombosis (PVT) is a rare and serious complication that affects prosthetic heart valves (PHVs). PVT consists in a thrombus or pannus formation that may hinder the leaflet movement, increasing the hydraulic resistance of the valve. In bileaflet mechanical valves (BMVs) in particular, the thrombus can involve either one leaflet or both, up to complete valvular obstruction. The overall incidence of PVT can vary from $0.5 \%$ to $6 \%$ per patient-year, with higher frequency in mitral position [1]. The mortality associated with PVT is approximately $10 \%$ [2].

Transthoracic Doppler echocardiography is a standard technique used after valve replacement for periodic monitoring of cardiac conditions, including prosthetic valve function. As for native cardiac valves, the mean pressure gradient (MPG) is the diagnostic index used to evaluate BMV function and to diagnose stenotic conditions. The MPG is the time-averaged transvalvular pressure drop along the systolic (for aortic valves) or diastolic (for mitral valves) period, estimated from blood flow peak velocity using the simplified Bernoulli formula [3]. MPG values above a critical threshold are considered an index of BMV. However, clinical studies [4,5] revealed that the MPG may result in false negatives in the detection of PVT of BMVs, a phenomenon named as Doppler silent thrombosis (DST).

From a clinical perspective, the relatively high incidence of DST and the potential failure of Doppler echocardiography in accurately diagnosing some PVT cases highlights the need for understanding the reasons of DST and, hence, to identify how to achieve higher sensitivity of Doppler ultrasound in the diagnosis of PVT. In this context, we hypothesized that MPG underestimation may be due to disturbances affecting the transvalvular blood flow, which could hamper the correct measurement of peak velocities. Such disturbances would characterize highly asymmetric stenotic configurations of the BMV. 
To test this hypothesis, we exploited computational fluid-dynamic (CFD) analyses, which allow to compute the detailed velocity and pressure fields also in complex fluid domains and in presence of flow disturbances.

Of note, the numerical simulation of blood flow through a BMV represents a complex fluid-structure interaction (FSI) problem, where a moving solid structure (valve leaflets) strongly interacts with the surrounding fluid (blood). Moreover, the motion of the valve leaflets is characterized by rapid and large rotations (by up to about $60^{\circ}$ ), which results in a challenging numerical problem to perform. These technical challenges, along with the interest for quantifying the function of BMVs and their effect on blood, have motivated several studies on the FSI modeling of BMVs. Currently a broad literature exists, which presents studies that are heterogeneous from several standpoints. First, the approaches used to impose FSI, either through moving methods, as for the Arbitrary Lagrangian Eulerian (ALE) method [6-10], or through fixed grid methods consisting in evolutions of the Immersed Boundary (IB) method [11-13]. Second, the site of simulated BMV implantation, which is the aortic position in the vast majority of the published studies $[7,8,14-17]$, and is the mitral position only in few studies [18]. Third, the aim of the numerical analysis, which can range to the highly detailed quantification of shear stresses exerted on blood flowing through the valve as a mean to assess the risk of mechanically induced blood damage [16] to the assessment of intraventricular fluid dynamics associated to the presence of a BMV either in aortic [19] or mitral [18] position. In this work, we exploited our previously implemented ALE approach [7-9] to simulate the fluid dynamics through a commercial BMV in mitral position, in normal conditions and in two pathological conditions with the same level of stenosis, and with the aim of assessing possible accuracies in Doppler echocardiography that may lead to DST. To this aim, we implemented a post-processing approach replicating the acquisition and processing of Doppler echocardiography. We then compared our Doppler-like pressure drop data i) vs. in vitro experimental data obtained with an experimental campaign designed to validate the computational model, to test the reliability of 
model predictions and ii) vs. pressure drop values directly yielded by the CFD analyses to test our working hypothesis.

\section{MATERIALS AND METHODS}

\section{The modeled BMV}

The Sorin Bicarbon Fitline size 25mm (Sorin Group SpA, Mirandola, Italy, Fig.1.A-D) in mitral position was modeled. Three diastolic configurations of the valve were considered in experimental tests and FSI models (Fig. 1.E-G):

- well-functioning (N60), with leaflet opening angles of $60^{\circ}$ and a $239.5 \mathrm{~mm}^{2}$ orifice area, computed as the projection of the valve lumen on the cross-section plane passing through the valve hinges;

- stenotic symmetric (SS35), with a $35^{\circ}$ opening angle for both leaflets and a $114.6 \mathrm{~mm}^{2}$ orifice area;

- stenotic asymmetric (SA57), with a $57^{\circ}$ opening angle for one leaflet, with the second leaflet completely closed, and a $114.6 \mathrm{~mm}^{2}$ orifice area;

\section{Experimental tests}

In vitro tests on the BMV were run on a previously described pulsatile mock loop [20], adapted for this experimental campaign. The mock loop includes an atrial chamber located downstream of a reservoir, a valve housing and a ventricular chamber. The latter is connected to a pulsatile computer-controlled piston pump that generates physiological flow rate waveforms; a one-way service valve connects the ventricular chamber to an RCR afterload circuit, designed to replicate the impedance of the systemic circulation (Fig. 2). A flow straightener is located the inlet of the atrial chamber, where the flow enters transversally with respect to the main flow direction through the valve. In the ventricular chamber, 1-mm-diameter stainless-steel bars with adjustable position acted as stoppers for limiting the motion of the BMV leaflets, thus replicating the SS35 and SA57 
configurations.

For each BMV configuration, a physiological transmitral flow rate was imposed. The diastolic transmitral flow rate was based on the tracing of a continuous wave Doppler (Fig.3) obtained during routine follow-up in a stable patient with a Sorin Bicarbon Fitline implanted in mitral position. The flow rate time-course was obtained by multiplying the Doppler-derived velocity by the effective orifice area of the valve. The systolic (transaortic) flow rate was approximated by a quadratic function, obtained by assuming a cardiac frequency of 75 beats/minute and a cardiac output equal to the diastolic filling volume. The transvalvular mitral pressure drop $(\Delta \mathrm{P})$ throughout the diastolic period was measured by two piezoresistive transducers (143PC05D, Honeywell Inc, Morristown, NJ) placed upstream from and downstream of the valve. The working fluid consisted in a solution of distilled water and glycerol (in the proportion $70 / 30 \mathrm{v} / \mathrm{v}$ ) that replicates the rheological properties of blood at $37^{\circ} \mathrm{C}$ [21]. Experimental data were acquired by a A/D converter (DAQ USB 6210, National Instruments, Austin, TX) at a sampling frequency of $200 \mathrm{~Hz}$. For each BMV configuration, the MPD was obtained by averaging the data from 5 consecutive cardiac cycles. Data are reported as mean $\pm S D$.

\section{Computational models and simulation settings}

The fluid dynamics across the BMV in each configuration was simulated with the finite volume solver ANSYS Fluent v14.5 (Ansys Inc, Irvine, CA, USA) using the grid deformation method integrated with a strongly-coupled FSI code based on the ALE formulation, developed by our group [9].

The geometry of the valve (valve leaflets and annular ring) was obtained from technical drawings provided by the manufacturer (Fig.1.A-D). The fluid domain was defined at the beginning of the diastolic phase (i.e., with the valve in closed configuration) through Design Modeler (Ansys Inc, Irvine, CA, USA). It included the relevant parts of the in vitro set-up: the valve housing, the atrial and ventricular chambers, an inlet conduit transversal to the atrial chamber and an outlet conduit at one side of the ventricular chamber, corresponding to the piston-pump connection in the 
experimental set-up (Fig.4). The fluid domain was discretized in $4.510^{6}$ tetrahedral elements in Meshing (Ansys Inc, Irvine, CA, USA): the mesh was finer $(0.4 \mathrm{~mm})$ at the valve housing and immediately downstream of it, and larger in the remainder of the fluid domain $(1 \mathrm{~mm})$.

The flow straightener between the inlet conduit and the atrial chamber was modeled as a porous material with permeability equal to $2.1310^{-10} \mathrm{~m}^{2}$ and inertial coefficients equal to $172271 \mathrm{~m}^{-1}$, based on experimental characterizations. The working fluid was modeled as Newtonian and incompressible with isothermal rheological properties of blood at $37^{\circ} \mathrm{C}$ (a density of $1060 \mathrm{~kg} / \mathrm{m}^{3}$ and a dynamic viscosity of $0.003 \mathrm{~Pa}$ s).

The same transmitral flow rate of the experimental tests was imposed at the inlet of the fluid domain and a zero constant pressure was set at the outlet. Non-deformability and no slip conditions were applied to the other boundaries of the fluid domain, except the valve leaflet surfaces that were assigned moving boundary conditions and modeled as rigid bodies with one degree of freedom, i.e., the rotation around the axis connecting the hinges. The angular acceleration of the leaflets deriving from the local distribution of hydrostatic pressure was computed through user-defined functions and the leaflet angular position was updated during the simulation.

At each time step the fluid and the structure were solved sequentially, and a fully coupled approach was implemented by iterating the time step until convergence of the numerical solutions of both the rigid body dynamics and the fluid field. The three opening configurations of the valve (N60, SS35 and SA57) were controlled by means of kinematic constraints: leaflet angular velocity was set to zero when the maximum opening angle was reached. More details on the FSI algorithm are available in Appendix A.

Simulations were run with a time step of $0.02 \mathrm{~ms}$ for the entire cardiac cycle $(0.8 \mathrm{~s})$. The segregated solver was used to solve the governing equations of the fluid, the second order upwinding scheme was used for the space-discretization of the momentum equation, and the SIMPLE algorithm was 
used to couple pressure and velocity. A dynamic mesh zone was assigned in the valvular region to refine the grid accordingly with the motion of the leaflets [22].

\section{Computational data post-processing}

Trans-valvular $\Delta \mathrm{P}$ was obtained through two approaches. In the first approach, two planes immediately upstream from and downstream of the valve were considered. At each time-step, the space average of the pressure was computed over the two planes, yielding $P_{i \_u p}$ and $P_{i_{-} \text {down }}$, respectively. The mean transvalvular pressure drop ( $M P G_{\text {direct }}$ ) was computed as:

$$
M P G_{\text {doppler-like }}=\frac{\sum_{i}\left(P_{i \_u p}-P_{i \_d o w n}\right)}{N}
$$

Where $\mathrm{N}$ is the number of time-steps in the diastolic phase.

In the second approach, the Doppler ultrasound inspection of the flow field was mimicked. The Doppler sampling volume (SV) was modeled as a slice of $1 \mathrm{~mm}$ thickness aligned with the main flow direction (axial with respect to the valve). Different possible acquisition locations were investigated: for each valve configuration, one SV was located in the centerline of the central orifice, and another SV was located in the centerline of the lateral orifice (Fig.5). For each acquisition location, the axial velocity values of the grid cells falling into the SV were acquired at each time step and postprocessed in Matlab (The MathWorks, Inc., Natick, MA, USA) as follows.

At each i-th time step of the diastolic phase, the instantaneous transvalvular pressure drop $\left(\Delta P_{i}\right)$ was computed according to the simplified Bernoulli formula:

$$
\Delta P_{i}=4 v_{i_{-} \max }^{2}
$$

where $v_{i \_ \text {max }}$ is the peak axial velocity found in the SV at the i-th time step.

The mean transvalvular pressure drop ( $\left.M P G_{\text {doppler-like }}\right)$ over the diastolic period was then calculated as the arithmetic average of the computed $\Delta P_{B i}$

$$
M P G_{\text {doppler-like }}=\frac{\sum_{i} \Delta P_{i}}{N}
$$


Where $\mathrm{N}$ is the number of time-steps. Moreover, a histogram plot (called Doppler histogram) (Fig. 9) was obtained based on velocity values computed at the cells falling into the SV. To this aim, at every time-point velocity data were processed through the following steps:

- for every cell into the SV, the axial component of velocity were stored in a file;

- for the exported velocities, a histogram was built, which represented the discrete probability function of the axial velocity in the SV;

- each bar of the histogram was color-coded in greyscale: the higher bar, i.e., the higher the odds of the corresponding velocity value, the brighter the bar;

- the bars were "superimposed", so that for each one only the tip was visible with the corresponding level of brightness. As a result, a single column of pixels was obtained, where each pixel corresponded to a velocity value.

\section{RESULTS}

\section{FSI simulations}

The fluid-dynamic fields of the three valve configurations were evaluated during the diastolic period, with a focus on the maximally open configuration of the BMV.

Velocity field - In Fig.6, the N60 model at different opening instants is shown, together with the contours of velocity magnitude on a longitudinal plane. The peak velocity $(\sim 2 \mathrm{~m} / \mathrm{s})$ is within the range reported in the guidelines for the management of PHVs in mitral position [3]. The flow through the valve is well distributed in the three orifices, determining the typical triple-jet structure of bileaflet MVs [23], with comparable velocities in the three jets (Fig.7A) that start developing at the inflow of the valve.

In the SS35 configuration a triple-jet structure is also observed, but the jets are less homogeneous: in the central jet the peak velocity is about $3.2 \mathrm{~m} / \mathrm{s}$; in the lateral jets, which develop at the level of the leaflet tips, downstream with respect to the central jet, peak velocities are equal to about 1.7 $\mathrm{m} / \mathrm{s}$, i.e., are comparable to those computed in the $\mathrm{N} 60$ configuration (Fig. 8A and Fig.7B). 
In the SA57 configuration only two jets are identified, which develop from a region slightly upstream from the tip of the free leaflet. High velocity jets are present in the central $(\sim 2.5 \mathrm{~m} / \mathrm{s})$ and in the free lateral ( $\sim 3 \mathrm{~m} / \mathrm{s}$ ) orifices (Fig. 8.B Fig.7C).

Transvalvular $\Delta P$ - An example of Doppler-like histogram obtained from FSI simulations is shown in Fig.9, with reference to the lateral orifice of the SA57 configuration. MPG values obtained from such histograms are summarized in Table 1 for every BMV configuration. In the N60 configuration, the pressure drop is below the cutoff value $(8 \mathrm{mmHg})$ used to discriminate normal from pathological conditions [24], regardless of the approach used to compute the MPG. Both $M P G_{\text {direct }}$ and $M P G_{\text {doppler- }}$ like data well agree with clinical data published by Erdil and co-authors [25], obtained by echoDoppler on 40 stable subjects with implanted Sorin Bicarbon Fitline 25 in mitral position, where MPG values of $6.5 \pm 2.7 \mathrm{mmHg}$ (mean value \pm standard deviation) were reported. In configurations SA57 and SS35 data are increased by approximately a factor of 3 and 4, respectively, as compared to the N60 configuration, and are well above the $8 \mathrm{mmHg}$ cutoff value. $\mathrm{MPG}_{\text {doppler-like }}$ data obtained at the central and lateral orifices differ by $0.54,2.24$ and $2.83 \mathrm{mmHg}$ for configurations N60, SS35 and SA57, respectively. At both jets, data slightly overestimated the corresponding MPG direct $_{\text {values. }}$

\section{In vitro tests}

Experimental and computational data for MPG are reported in Table 1. Experimental MPGs was $5.6 \pm 0.1 \mathrm{mmHg}$ (N60 configuration), $18.1 \pm 0.5 \mathrm{mmHg}$ (SS35) and $22.1 \pm 0.6 \mathrm{mmHg}$. The general trend of these MPGs was consistent with data from the computational model, with the highest pressure drop for SA57 configuration. More in detail, the MPG direct underestimate the experimental data (maximum percentage deviation by $-16.6 \%$ obtained for the $\mathrm{N} 60$ configuration, minimum maximum percentage deviation by $-6.7 \%$ for the SA57 configuration). Comparing MPG doppler-like to experimental data, maximum deviation was $13.9 \%$ (N60, MPG doppler-like central orifice), minimum was $-0.2 \%$ (MPG doppler-like lateral orifice).

\section{DISCUSSION}


PVT is a rare (0.2\% to $6 \% /$ patient-year for left-sided prostheses) [26] but potentially life-threatening complication of heart valve replacement. Noninvasive tests for diagnosing mitral PVT include transthoracic, transesophageal echocardiography and fluoroscopy [27]. Transthoracic echocardiography and Doppler may accurately diagnose PVT in a large majority of cases based on high MPG, while transesophageal echocardiography may complete or confirm the diagnosis by giving a more accurate anatomic evaluation. However, in approximately $25 \%$ of cases with bileaflet MVs the diagnosis may be inaccurate by Doppler-derived MPG alone because patients may have normal or close-to-normal $\Delta \mathrm{P}$ despite abnormal motion of valve leaflets, which may only be accurately evaluated by fluoroscopy. Among these cases, the DST cases, i.e., the false negatives, are of particular concern and highlight the need to understand the reason for the inaccuracies of some diagnoses based on Doppler ultrasound imaging.

Doppler ultrasound MPG estimations are based on velocity measurements and on the use of the simplified Bernoulli formula, which is strictly valid for an inviscid flow. Hence, MPG estimations strongly depend on i) the reliability of the assumptions underlying the Bernoulli formula in a given scenario and ii) the correct measurement of velocities through a manual and operator-dependent procedure.

On this basis, we exploited in vitro modeling and FSI modeling to assess the function of a real BMV in three fully controlled conditions, characterized by a normal open configuration of the BMV and by two stenotic open configurations, which are expected to induce different derangements of the transvalvular flow field despite having the same degree of lumen reduction. The experimental apparatus used for the tests was rather simplified with respect to the in-vivo scenario, which made the local fluid dynamics different from the physio-pathologic one. Specifically, a large rigid box was used as the ventricular chamber, and auxiliary elements were embedded in the ventricular volume (far enough from the fluid dynamic region of interest) for repeatable leaflet control. Indeed, it should be considered that the experimental study was designed to validate the computational 
method. For this reason, the main goal of the tests was to obtain well repeatable and controllable experimental conditions that could be replicated by a computational model. In the in vitro tests, the MPG was obtained by the direct measurement of the pressure immediately upstream from and downstream of the BMV. In the FSI simulations, the same direct quantification was performed, and a Doppler-like measurement of the MPG was also obtained through an ad hoc processing procedure. The reliability of the FSI simulations in the context of the goals of the present study was confirmed by the very good agreement of all the computational MPG data with their in vitro counterpart.

Also, for the normally functioning BMV in particular, MPG data well agreed with in vivo measurements reported on a relatively large population of BMV recipients with no BMV stenosis. The virtual replica of the Doppler ultrasound analysis applied on the velocity field yielded by the FSI simulations yielded MPG values that were comparable to the corresponding direct measurement, independently of the considered BMV configuration and of the analyzed orifice. Differences between $M P G_{\text {doppler-like }}$ data obtained at the central and lateral orifices were larger as configurations with more evident derangements in the flow field were considered. Yet, these differences were in any case smaller than $3 \mathrm{mmHg}$. This result is consistent with previous in vitro findings on the sensitivity of Doppler measurements to the acquisition location [28], and it suggests that even in case of relevant flow derangements the Doppler-based measurement is moderately sensitive to the position of the probe; as a result, the uncertainty in the measurement may be relevant to DST only in patients with MPG values very close to the $8 \mathrm{mmHg}$ cutoff value, i.e., with very mild stenosis. This may explain why DTS patients may be frequently asymptomatic. These patients are likely those characterized by such almost normal MPG gradients and detection of disk motion anomalies is mandatory.

Also, it should be noted that in our virtual replica of the Doppler ultrasound analysis we assumed the sampling volume always aligned with the direction perpendicular to the BMV orifice plane. In other words, we assumed the ultrasound probe always optimally aligned with the transvalvular flow 
field. In the real examination, a tilted position of the probe could increase the uncertainty of the measurement.

\section{Limitations and future developments}

Two main simplifications were assumed in the study need and need to be mentioned.

The first simplification is in our FSI algorithm: the torque exerted on the prosthetic leaflets by the fluid flow is computed by accounting only for the effects of pressure and neglecting the viscous moment generated by the shear stresses acting on the leaflet surface, whereas previous studies on the FSI modeling of bi-leaflet mechanical valves have accounted for both effects [29]. Yet, is should be noted that this simplification is acceptable, especially in relation to the goals of the present study, because of two reasons: first, shear stresses acting on the atrial and ventricular side of the leaflets are smaller by two orders of magnitude than the pressure acting on those surfaces. This evidence was verified focusing on the simulated stenotic conditions, i.e., the ones where higher shear stresses could be expected, both during transient opening of the BMV leaflet and at peak flow rate. Second, owing to the curvilinear profile of the prosthetic leaflets, the lever arm available to shear stresses to exert a torque on the leaflets can be up to $2.3 \mathrm{~mm}$ on the ventricular side and $3.3 \mathrm{~mm}$ on the atrial side, whereas the lever arm available to pressure loads can be up to approximately $10.5 \mathrm{~mm}$. As a result, the contribution of shear stress to generating torque was indeed negligible. Besides, this evidence confirms the results we obtained in previously published analyses on BMVs [8].

The second simplification consists in the time-dependent flow rate imposed in the in vitro experiment and replicated in the simulations: a physiological transvalvular flow rate was used to simulate both normal and stenotic configurations. This assumption may be inaccurate, since BMV recipients may have reduced or altered cardiac frequency: hence they me have non-physiological trans-mitral flow rate values and waveforms. Of course, a major change in the flow rate would lead to a major change in the peak blood velocities through the BMV, and hence to different values of MPG as estimated through Doppler ultrasound. It is worth stressing that also in the real clinical 
setting the flow rate is typically unknown, since Doppler measurements only yield the time-course of blood velocity in the region inspected by the ultrasound beam. This lack of information may be more relevant than the uncertainty affecting the manual positioning of the ultrasound probe.

Accordingly, for the next future we plan to systematically investigate a reasonably wide range of different clinical scenarios with different BMV configurations combined with different flow rate conditions, also related to cases of PVT with concurrent atrial fibrillation or low stroke volume.

\section{ACKNOWLEDGEMENTS}

None

\section{NOMENCLATURE}

ALE=Arbitrary Lagrangian-Eulerian

BMV=Bileaflet Mechanical Valve

CFD=Computational Fluid Dynamics

DST=Doppler Silent Thrombosis

FSI=Fluid-structure Interaction

MPG=Mean pressure gradient

PHV=Prosthetic Heart Valve

PVT=Prosthetic Valve Thrombosis

SV=Sampling volume

\section{APPENDIX A - FSI interaction algorithm}


Each MV leaflet was modeled as a rigid body with one degree of freedom (the rotation around the axis connecting the hinges), governed by the Euler's second law for rigid bodies

$$
M=I \ddot{\theta}
$$

where $I$ is the body moment of inertia about the rotation axis, equal to $7.8110^{-9} \mathrm{~kg} \mathrm{~m}^{2}, \ddot{\theta}$ is the angular acceleration of the body and $M$ is the torque acting on the rigid body due to external forces. In this case, the external forces are generated by the fluid dynamic field on the leaflet surface (gravity contribution is neglected) and result from hydrostatic pressure and shear stresses. In the specific case of the valve leaflets, the contribution of the shear stress is negligible with respect to the hydrostatic contribution, in fact the shear stress contribution to the torque is associated to small lever arms. For this reason, in the computation of the torque only the pressure forces were considered and $M$ was defined as follows

$$
M=\iint_{\Gamma} p \vec{n} \times \overrightarrow{\mathrm{r}} d A
$$

where $\Gamma$ is the external surface of the leaflet, $p$ is the pressure acting on the infinitesimal surface area $d A, \vec{n}$ is the normal vector of $d A$ and $\vec{r}$ is the lever arm associated to the infinitesimal force $p \vec{n} d A$.

In the computational models Eq. 5 was approximated by the summation of the discrete contributions computed over the faces of the grid cells belonging to the leaflet surface

$$
M=\sum_{f} p_{f} \overrightarrow{A_{f}} \times \overrightarrow{\mathrm{r}_{f}}
$$

where $p_{f}$ is the pressure of the fluid in the center of face $f, \overrightarrow{A_{f}}$ is the face area vector, which is defined as the vector normal to the face with a modulus equal to the face area and pointing outward of the fluid domain, $\overrightarrow{\mathrm{r}_{f}}$ is the lever arm with respect to the rotation axis computed at the center of the face. The angular acceleration can then be computed from the torque $M$ as in Eq. 4. 
At each time step the fluid and the structure are solved sequentially, and the strongly coupled interaction is implemented by iterating the same time step until convergence in the numerical solution of both the rigid body dynamics and the fluid field is achieved.

The convergence of the dynamics of the rigid bodies is considered satisfied when the difference between the angular accelerations of two consecutive iterations of the same time step falls below a tolerance $\varepsilon_{\text {threshold }}$, which was set equal to $500 \mathrm{~s}^{-2}$ in our calculations as in $[7,8]$. More in details, the implemented FSI algorithm performed as follows [8]:

- at the i-th time step and k-th FSI iteration, the solver first updates the leaflet position computed from a trial acceleration value $\ddot{\theta}_{i}^{k *}$. Then, the flow field is solved until convergence (giving velocity and pressure in the fluid domain $v_{i}^{k}, p_{i}^{k}$ ), the torque acting on each leaflet $\left(M_{i}^{k}\right)$ is computed and the k-th value of the acceleration is calculated $\ddot{\theta}_{i}^{k}$

- the convergence is checked by computing a residual value for the k-th iteration

$$
\varepsilon_{i}^{k}=\ddot{\theta}_{i}^{k *}-\ddot{\theta}_{i}^{k}
$$

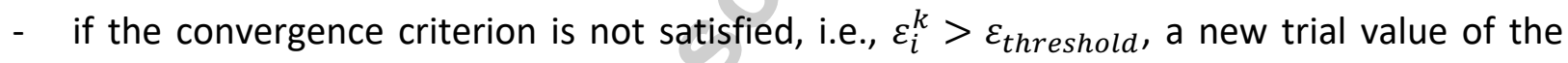
acceleration is computed for the current time step using an under-relaxation factor $(\omega)$

$$
\ddot{\theta}_{i}^{k+1 *}=\ddot{\theta}_{i}^{k}+\omega_{1} \varepsilon_{i}^{k}
$$

and the time step is re-iterated. For a better stabilization of the solution, $\omega_{1}$ is set to 0.2 if $\varepsilon_{i}^{k}$ and $\varepsilon_{i}^{k-1}$ have the same sign, and to 0.1 if $\varepsilon_{i}^{k}$ and $\varepsilon_{i}^{k-1}$ have opposite signs indicating that the solution is oscillating around a convergence value;

- if the convergence criterion is satisfied, i.e., $\varepsilon_{i}^{k}<\varepsilon_{\text {threshold, }}$, then the $\mathrm{i}$-th time step has converged, the solution at the i-th time step is stored

$$
\ddot{\theta}_{i}=\ddot{\theta}_{i}^{k}, \quad v_{i}=v_{i}^{k}, \quad p_{i}=p_{i}^{k}
$$

and the subsequent time step $(\mathrm{i}+1)$ starts with a trial value of acceleration computed as

$$
\ddot{\theta}_{i+1}^{1 *}=\ddot{\theta}_{i}+\omega_{2} \varepsilon_{i}^{k}
$$


where $\omega_{2}$ is set to 0.08 .

At each FSI iteration, the angular velocity $\left(\dot{\theta}_{i}^{k}\right)$ and the leaflet angular position $\left(\theta_{i}^{k}\right)$ are computed by integrating the angular acceleration as follows

$$
\begin{gathered}
\dot{\theta}_{i}^{k}=\dot{\theta}_{i-1}+\ddot{\theta}_{i}^{k} \Delta t \\
\theta_{i}^{k}=\theta_{i-1}+\dot{\theta}_{i}^{k} \Delta t+\frac{1}{2} \ddot{\theta}_{i}^{k} \Delta t^{2}
\end{gathered}
$$

where $\Delta \mathrm{t}$ is the time step between time step $\mathrm{i}+1$-th and $i$-th.

Convergence within the single time step was achieved within two to ten iterations, where the number of required iterations increased with the angular acceleration characterizing the rigid motion of the prosthetic leaflets. 


\section{REFERENCES}

[1] Caceres-Loriga, F.M., Pérez-Lopez, H., Santos-Gracia, J., and Morlans-Hernandez, K., 2006, "Prosthetic heart valve thrombosis: Pathogenesis, diagnosis and management," Int. J. Cardiol., 110, pp. 1-6.

[2] Roudaut, R., Serri, K., and Lafitte, S., 2007, "Thrombosis of prosthetic heart valves: diagnosis and therapeutic considerations," Heart, 93, pp. 137-42.

[3] Zoghbi, W.A., Chambers, J.B., Dumesnil, J.G., Foster, E., Gottdiener, J.S., Grayburn, P.A., Khandheria, B.K., Levine, R.A., Marx, G.R., Miller, F.A., Nakatani, S., Quinones, M.A., Rakowski, H., Rodriguez, L.L., Swaminathan, M., Waggoner, A.D., Weissman, N.J., and Zabalgoitia, M., 2009, “Recommendations for Evaluation of Prosthetic Valves With Echocardiography and Doppler Ultrasound. A Report From the American Society of Echocardiography's Guidelines and Standards Committee and the Task Force on Prosthetic Valves, Developed in Conjunction," J. Am. Soc. Echocardiogr., 22, pp. 9751014.

[4] Montorsi, P., Cavoretto, D., Alimento, M., Muratori, M., and Pepi, M., 2003, "Prosthetic mitral valve thrombosis: can fluoroscopy predict the efficacy of thrombolytic treatment?," Circulation, 108, pp. II79-II84.

[5] Muratori, M., Montorsi, P., Teruzzi, G., Celeste, F., Doria, E., Alamanni, F., and Pepi, M., 2006, "Feasibility and diagnostic accuracy of quantitative assessment of mechanical prostheses leaflet motion by transthoracic and transesophageal echocardiography in suspected prosthetic valve dysfunction," Am. J. Cardiol., 97, pp. 94-100.

[6] Cheng, R., Lai, Y.G., and Chandran, K.B., 2004, "Three-dimensional Fluid-Structural Interaction Simulation of Bileaflet Mechanical Heart Valve Flow Dynamics," Ann. Biomed. Eng., 32, pp. 1471-1483. 
[7] Nobili, M., Morbiducci, U., Ponzini, R., Del Gaudio, C., Balducci, A., Grigioni, M., Maria Montevecchi, F. and Redaelli, A., 2008, "Numerical simulation of the dynamics of a bileaflet prosthetic heart valve using a fluid-structure interaction approach," J. Biomech., 41, pp. 2539-2550.

[8] Nobili, M., Passoni, G. and Redaelli, A., 2007, "Two fluid-structure approaches for 3D simulation of St. Jude Medical bileaflet valve opening," J. Appl. Biomater. Biomech., 5, pp. 49-59.

[9] Redaelli, A., Bothorel, H., Votta, E., Soncini, M., Morbiducci, U., Del Gaudio, C., Balducci, A. and Grigioni, M., 2004, "3-D Simulation of the st. Jude medical bileaflet valve opening process: Fluid-structure interaction study and experimental validation," J. Heart Valve Dis., 13, pp. 804-813.

[10] Souli, M., Ouahsine, A., and Lewin, L., 2000, “ALE formulation for fluid-structure interaction problems," Comput. Methods Appl. Mech. Eng., 190, pp. 659-675.

[11] Ge, L., and Sotiropoulos, F., 2007, "A numerical method for solving the 3D unsteady incompressible Navier-Stokes equations in curvilinear domains with complex immersed boundaries," J. Comput. Phys., 225, pp.1782-1809.

[12] Gilmanov, A., and Sotiropoulos, F., 2005, "A hybrid Cartesian/immersed boundary method for simulating flows with 3D, geometrically complex, moving bodies," J. Comput. Phys., 207, pp. 457-492.

[13] Peskin, C.S., 1972, "Flow patterns around heart valves: A numerical method," J. Comput. Phys., 10, pp. 252-271.

[14] Annerel, S., Claessens, T., Degroote, J., Segers, P., and Vierendeels, J., 2014, "Validation of a numerical FSI simulation of an aortic BMHV by in vitro PIV experiments," Med. Eng. Phys., 36, pp. 1014-1023.

[15] Annerel, S., Degroote, J., Claessens, T., Segers, P., Verdonck, P. and Vierendeels, J., 2012, 
"The upstream boundary condition influences the leaflet opening dynamics in the numerical FSI simulation of an aortic BMHV," Int. J. Numer. Method. Biomed. Eng., 28, pp. 745-760.

[16] Borazjani, I., Ge, L., and Sotiropoulos, F., 2010, "High-resolution fluid-structure interaction simulations of flow through a bi-leaflet mechanical heart valve in an anatomic aorta," Ann. Biomed. Eng., 38, pp. 326-344.

[17] De Beule, M., Mortier, P., Carlier, S.G., Verhegghe, B., Van Impe, R., and Verdonck, P., 2008, "Realistic finite element-based stent design: The impact of balloon folding," J. Biomech., 41, pp.383-389.

[18] Su, B., Kabinejadian, F., Phang, H.Q., Kumar, G.P., Cui, F., Kim, S., Tan, R.S., Hon, J.K.F., Allen, J.C., Leo, H.L., and Zhong, L., 2015, “Numerical modeling of intraventricular flow during diastole after implantation of BMHV," PLoS One, 10, pp. e0126315

[19] Votta, E., Le, T.B., Stevanella, M., Fusini, L., Caiani, E.G., Redaelli, A., and Sotiropoulos, F., 2013, "Toward patient-specific simulations of cardiac valves: State-of-the-art and future directions," J. Biomech., 46, pp. 217-228.

[20] Vismara, R., Pavesi, A., Votta, E., Taramasso, M., Maisano, F., and Fiore, G.B., 2011, “A pulsatile simulator for the in vitro analysis of the mitral valve with tri-axial papillary muscle displacement," Int. J. Artif. Organs, 34, pp. 383-391.

[21] Quaini, a., Canic, S., Glowinski, R., Igo, S., Hartley, C.J., Zoghbi, W., and Little, S., 2012, "Validation of a 3D computational fluid-structure interaction model simulating flow through an elastic aperture", J. Biomech., 45, pp.310-318.

[22] ANSYS FLUENT User's Guide Release 14.5, ANSYS, Inc., Southpointe, 275 Technology Drive, Canonsburg, PA 15317, 2012.

[23] Yoganathan, A.P., Chandran, K.B., and Sotiropoulos, F., 2005, "Flow in prosthetic heart valves: State-of-the-art and future directions," Ann. Biomed. Eng., 33, pp. 1689-1694. 
[24] Montorsi, P., Cavoretto, D., Parolari, A., Muratori, M., Alimento, M., and Pepi, M., 2002, "Diagnosing prosthetic mitral valve thrombosis and the effect of the type of prosthesis," Am. J. Cardiol., 90, pp. 73-76.

[25] Erdil, N., Cetin, L., Demirkilic, U., Tatar, H., and Uzun, M., 2003, "Experience of the Small Size $(25 \mathrm{~mm})$ Sorin Bicarbon Bileaflet Prosthetic Valve in Patients with Small Mitral Annuli," J. Card. Surg., 18, pp. 532-538.

[26] Horstkotte, D., and Burckhardt, D., 1995, "Prosthetic valve thormbosis," J. Heart Valve Dis., 4, pp. 141-153.

[27] Montorsi, P., De Bernardi, F., Muratori, M., Cavoretto, D., and Pepi, M., 2000, "Role of cine-fluoroscopy, transthoracic, and transesophageal echocardiography in patients with suspected prosthetic heart valve thrombosis," Am. J. Cardiol., 85, pp. 58-64.

[28] Evin, M., Guivier-Curien, C., Pibarot, P., Kadem, L., and Rieu, R., 2016, "Are the Current Doppler Echocardiography Criteria Able to Discriminate Mitral Bileaflet Mechanical Heart Valve Malfunction? An In Vitro Study," Artif. Organs, 40, pp. E52-60.

[29] Annerel, S., Degroote, J., Claessens, T., Dahl, S.K., Skallerud, B., Hellevik, L.R., van Ransbeeck, P., Segers, P., Verdonck, P., and Vierendeels, J., 2012a, "A fast strong coupling algorithm for the partitioned fluid-structure interaction simulation of BMHVs," Comput. Methods Biomech. Biomed. Engin., 15, pp. 1281-1312. 


\section{FIGURE LEGENDS}

Figure 1. Top Panel: Sorin Bicarbon Fitline size $25 \mathrm{~mm}$ as seen in a 3-D view (A), from the ventricle (B), and from two lateral views (C-D). Bottom Panel: sketch of the three valve configurations considered in the study: E) well-functioning valve (N60), F) dysfunctional symmetric stenosis (SS35), G) dysfunctional asymmetric stenosis (SA57). For each configuration, the opening angle of the leaflet is shown with respect to the closed configuration (dashed line).

Figure 2. Schematic of the experimental set-up composed by the systemic impedance simulator (S), the reservoir $(R)$, the programmable pump $(P)$ and the test bench $(T)$. Modified from the paper by Vismara and colleagues (Vismara et al., 2011).

Figure 3. Echo-Doppler signal used to derive the time-dependent flow rate used as inlet boundary condition in the FSI simulations.

Figure 4. 3D model of the test bench for the FSI analyses; the different components are indicated.

Figure 5. Location of the central (orange) and lateral (green) scanning volumes (SVs) used to process fluid dynamic data according to the Doppler-like approach in the three models.

Figure 6. Contours of velocity magnitude $(v)$ obtained during the opening phase $(A, B, C)$ and at peak diastolic flow (D) for the N60 configuration. The 3D geometry of the leaflets is shown.

Figure 7. Right panel. Plots of axial velocity profiles along a line crossing the valves as shown in the left panel. Results for the three configurations of valves are reported: A. N60, B. SS35, and C. SA57.

Figure 8. Contours of velocity magnitude (v) at peak diastolic flow for the SS35 (left) and SA57 (right) configurations. The 3D geometry of the leaflets is shown. The vertical white bars represent the structural elements used to limit valve rotation in the experimental in vitro tests.

Figure 9. Doppler histogram of the SA57 configuration obtained from the numerical simulations. 


\section{TABLES}

Table 1. Time-averaged transvalvular pressure drop (MPG) obtained from FSI analyses and from in vitro tests and for the three tested configurations. Computational results were computed directly as the difference between pressure values over two planes upstream from and downstream of the valve ( $\left.M P G_{\text {direct }}\right)$, and by mimicking the Doppler ultrasound examination of the transvalvular flow (MPGDoppler-like). Experimental values are reported as mean \pm standard deviation computed over 5 consecutive diastolic periods.

\begin{tabular}{ccccc} 
Configuration & \multicolumn{3}{c}{ Computational Results } & In vitro data \\
\hline & MPG $_{\text {direct }}[\mathrm{mmHg}]$ & \multicolumn{2}{c}{ MPG } & Doppler-like $[\mathrm{mmHg}]$ \\
& & Central Orifice & Lateral Orifice & MPG [mmHg] \\
\hline N60 & 4.67 & 6.38 & 5.84 & $5.6 \pm 0.1$ \\
SS35 & 15.48 & 19.95 & 17.71 & $18.1 \pm 0.5$ \\
SA57 & 20.63 & 24.89 & 22.06 & $22.1 \pm 0.6$ \\
\hline
\end{tabular}


A)

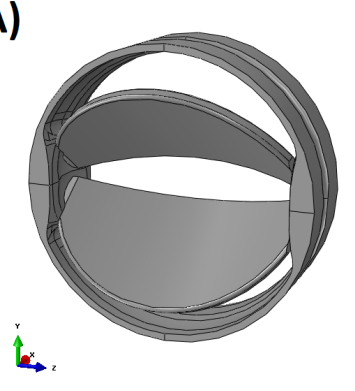

B)

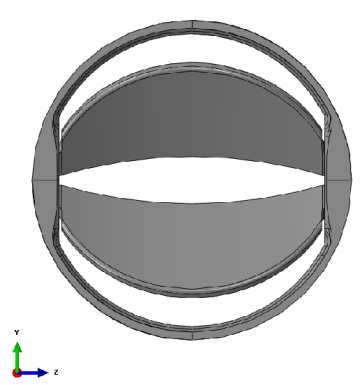

C)

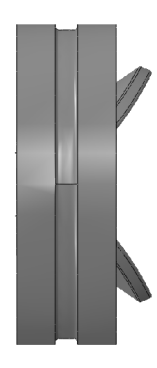

D)

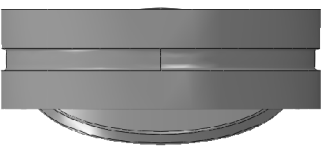

E)

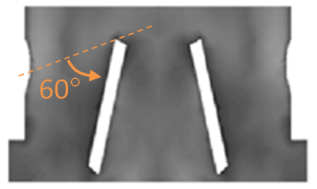

F)

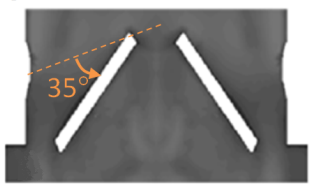

G)

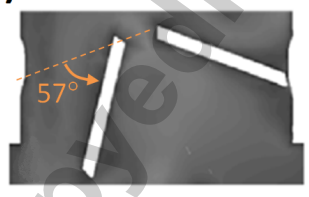

Figure 1 


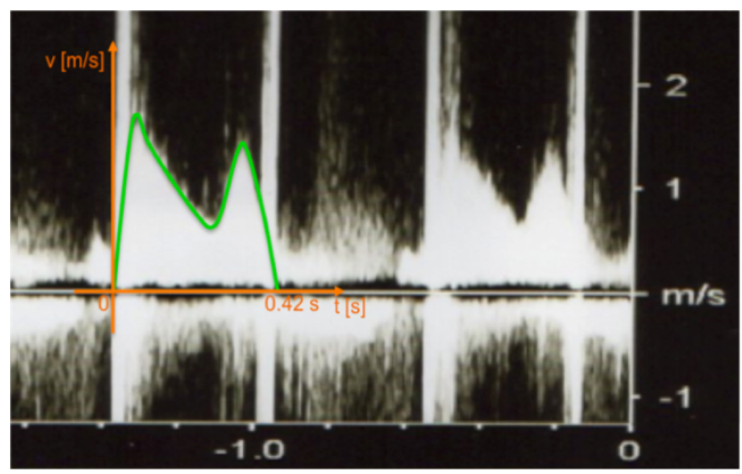

Figure 3

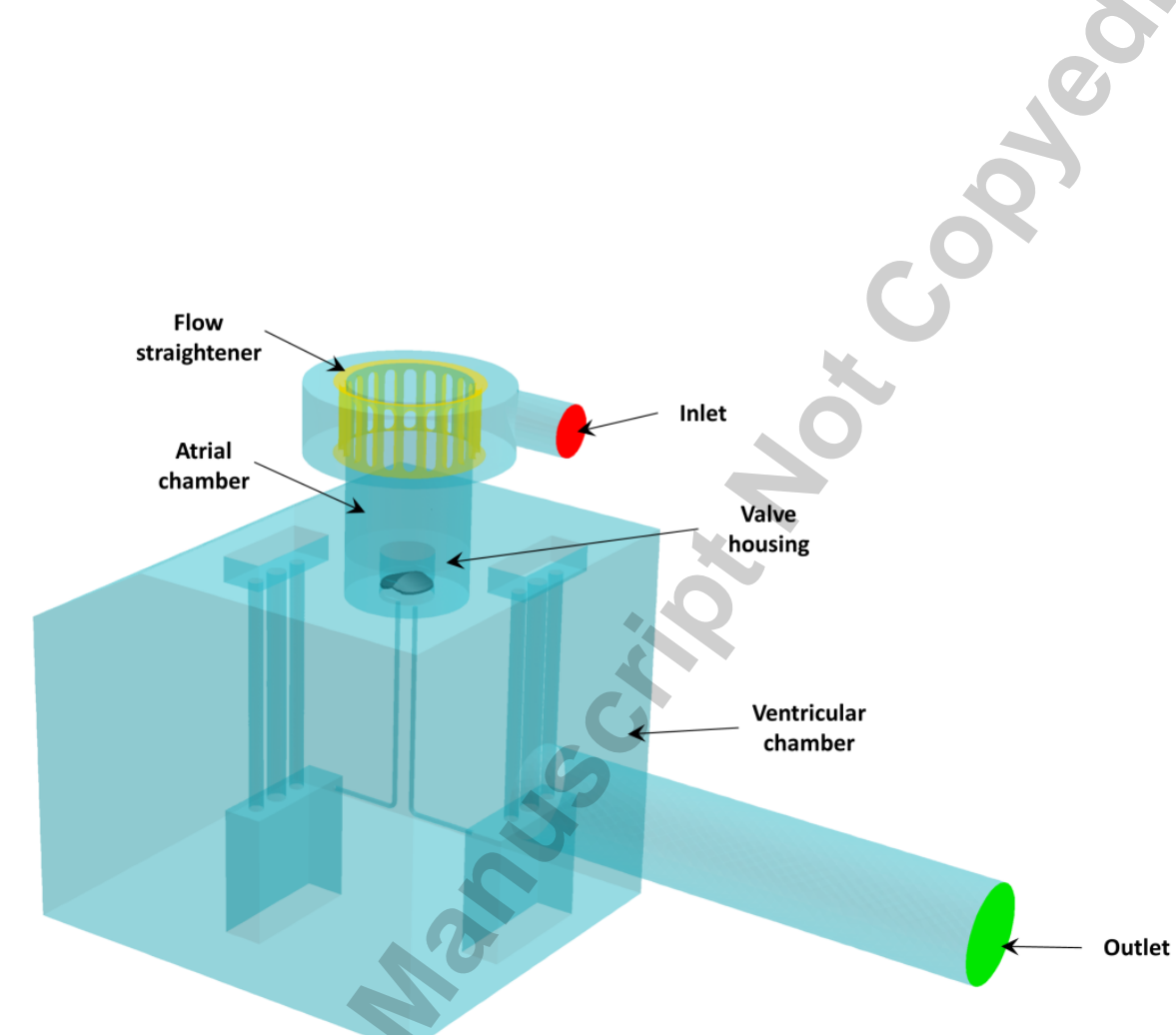

Figure 4
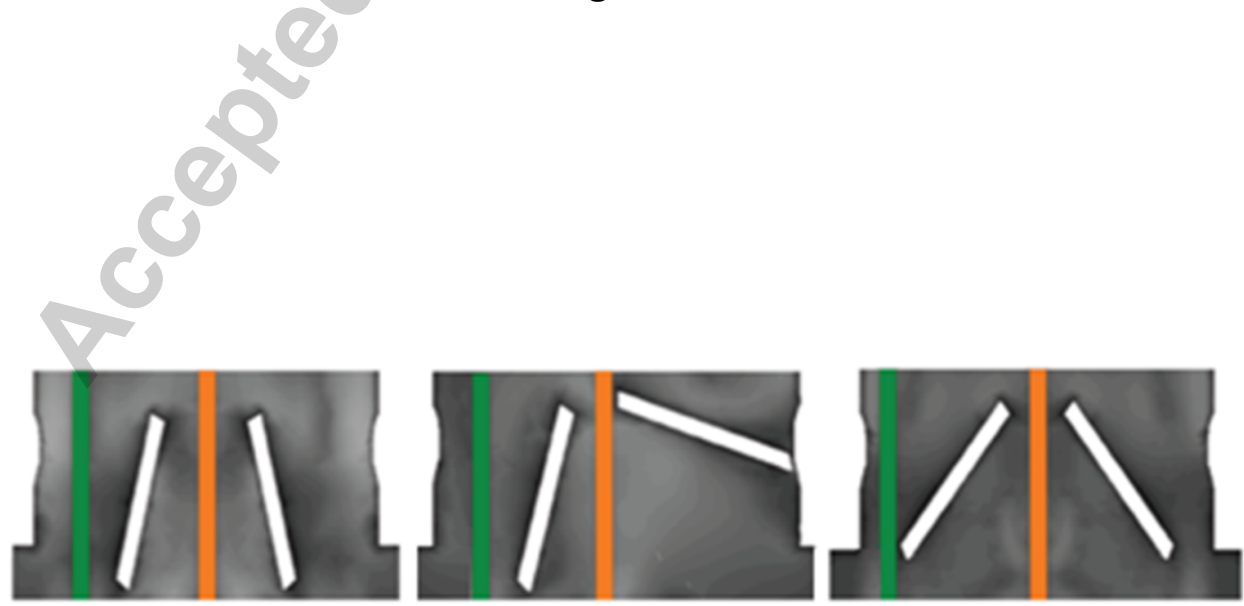

Figure 5 


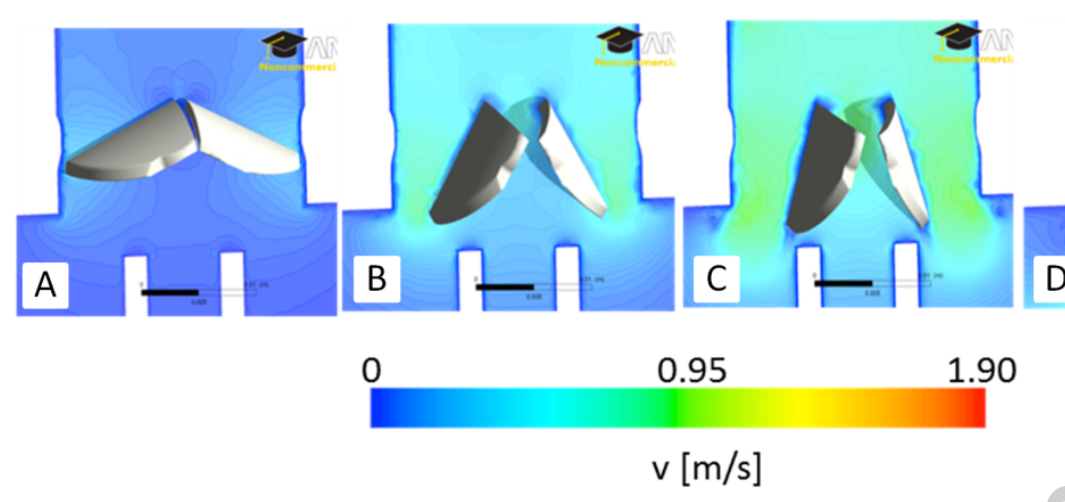

Figure 6

A

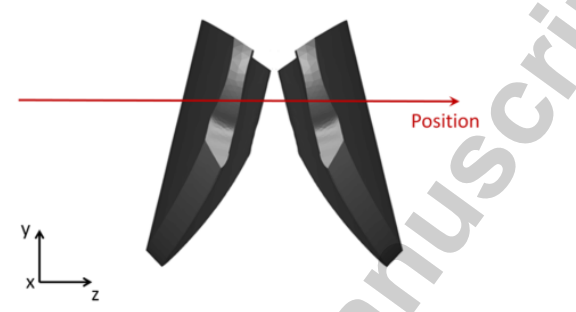

10
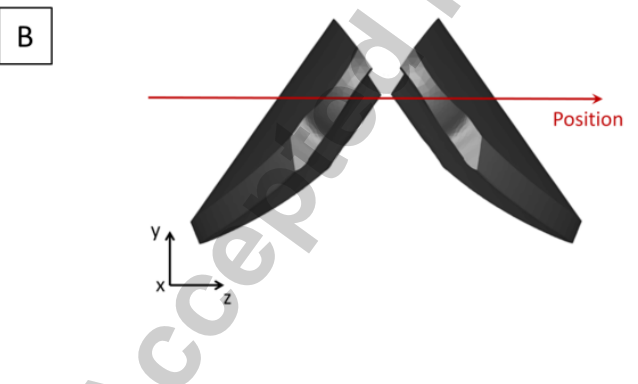

C

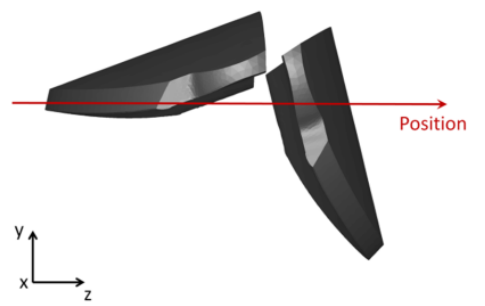

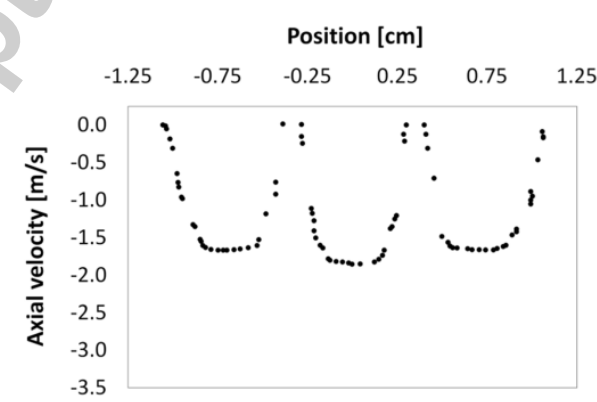

Position $[\mathrm{cm}]$

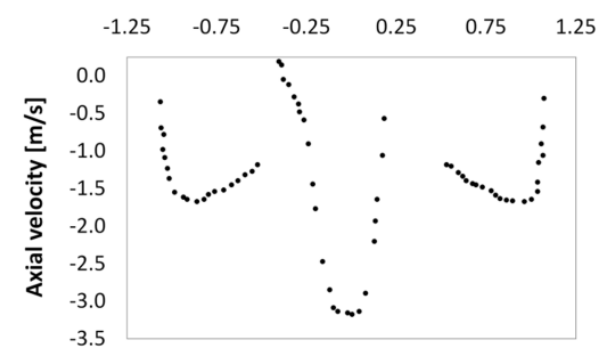

Position [cm]

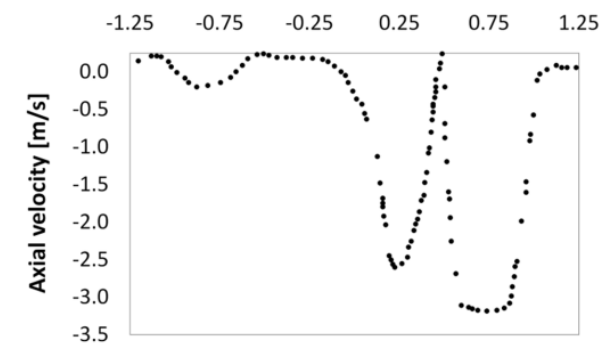

Figure 7 

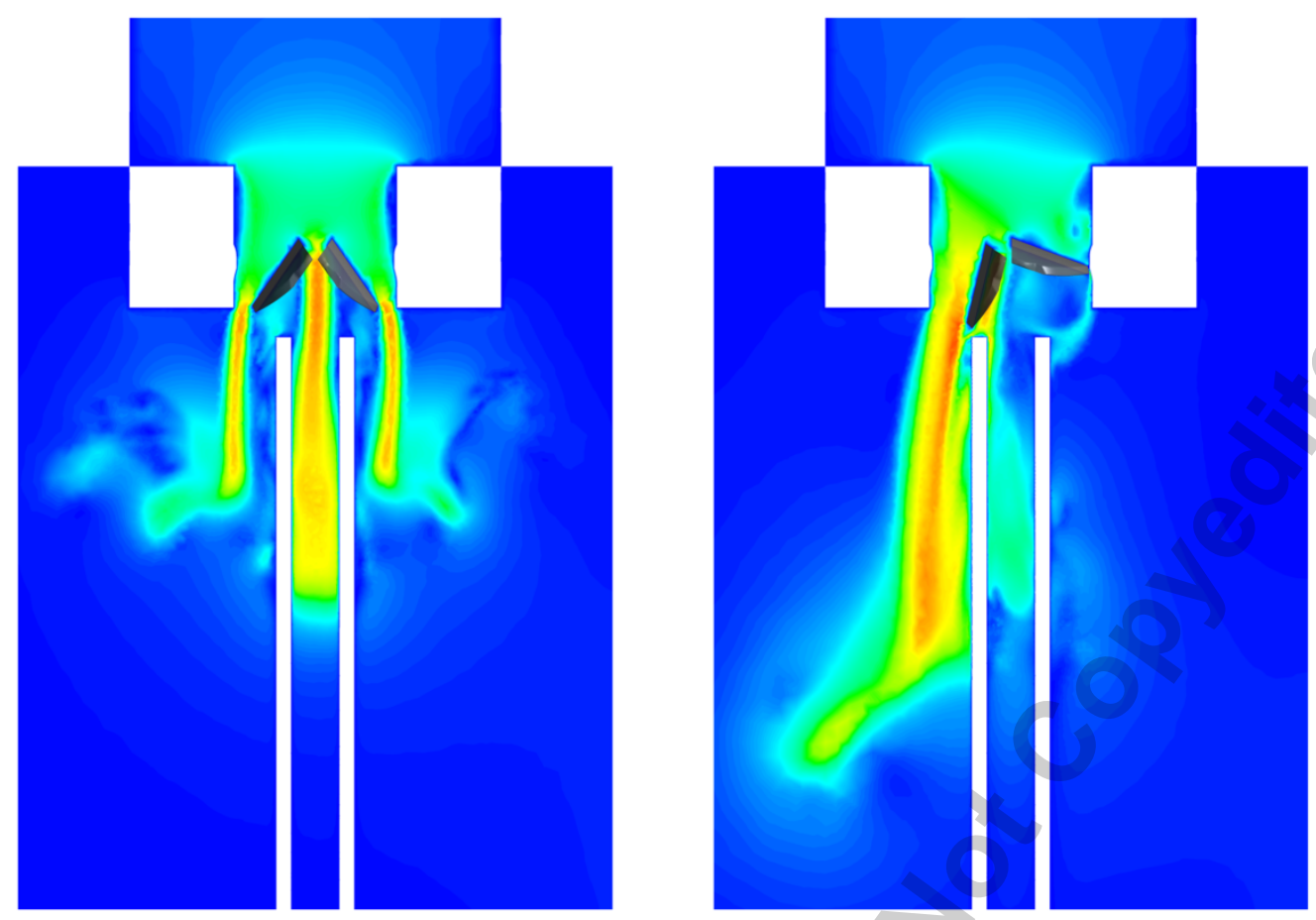

$\mathrm{v}[\mathrm{m} / \mathrm{s}]$

3.6

Figure 8

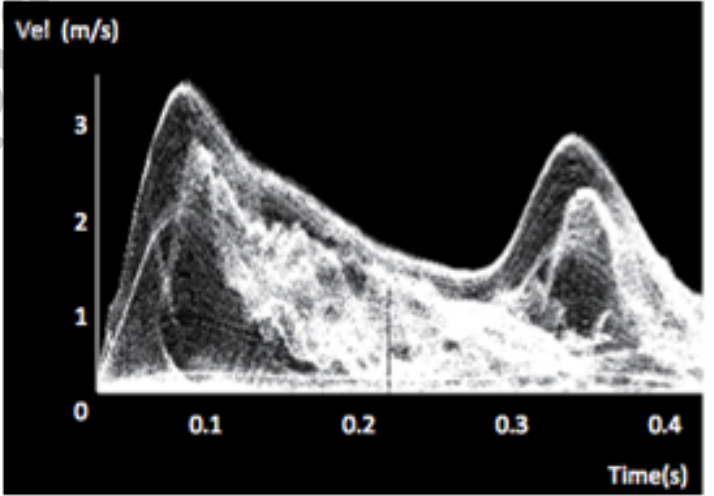

Figure 9 
